\title{
Modelo de capas para motores encapsulados de imán permanente con arranque en línea
}

\section{Layer Model for Canned Line-Start Permanent Magnet Motors}

\author{
Peralta-Sánchez Edgar \\ Universidad Popular Autónoma del Estado de Puebla \\ Departamento de Ingenierías \\ Correo:edgar.peralta@upaep.mx \\ Rodríguez-Rivas Jaime José \\ Instituto Politécnico Nacional \\ Escuela Superior de Ingeniería Mecánica y Eléctrica \\ Sección de Estudios de Posgrado e Investigación \\ Correo:jjrodriguezr@ipn.mx
}

\author{
Salazar-Amador María del Rubí \\ Universidad Popular Autónoma del Estado de Puebla \\ Departamento de Ingenierías \\ Correo: rubi.salazar@upaep.mx
}

Información del artículo: recibido: febrero de 2015, reevaluado: abril de 2015, aceptado: agosto de 2015

\section{Resumen}

En este artículo se describe el concepto de 'bomba encapsulada' y se desarrolla un análisis del motor encapsulado. El motor se integra en la bomba y es de imanes permanentes (IP) con arranque directo a la línea, cuenta con dos cápsulas de metal, una en el estator y otra en el rotor. La cápsula del rotor tiene doble función: actuar como elemento de inducción para el arranque directo a la línea y cubrir al rotor del líquido que se bombea. En el artículo se desarrolla un pseudomodelo analítico en 3D basado en la teoría de capas, el cual demuestra cómo el enfoque convencional para esta técnica puede modificarse para permitir adaptar los voltajes como alimentación de entrada y predecir la operación síncrona. El modelo se valida experimentalmente usando un prototipo de una motobomba de $10 \mathrm{~kW}$, y se utiliza para estudiar la influencia de determinadas características de diseño tales como: el espesor de la cápsula del rotor, la fuerza magnetomotriz de los imanes del rotor y la longitud del saliente de la estructura de la cápsula del estator.

\section{Descriptores:}

- motor de arranque directo

- motor de imanes permanentes de arranque en línea

- bomba química sin sello 


\begin{abstract}
This paper describes the concept of 'canned pump' and the analysis of the canned motor. The motor is integrated directly into the pump and operates as a line-start permanent-magnet motor. It has two cans one in the stator and the other in the rotor. The rotor containment can provide a dual function: acting as the induction element to provide the line-start capability and provides a protection to the rotor against the fluid being pumped. The paper describes the development of an analytical pseudo-3D model based on the layer theory and demonstrates how the conventional approach can be modified to accommodate voltage-forced solutions and synchronous operation. The model is validated experimentally using a $10 \mathrm{~kW}$ prototype pump motor and the model is used to examine the influence of certain design features such as the thickness of the rotor containment, the rotor magnets strength and the overhang length of the stator containment can.
\end{abstract}

\section{Introducción}

Los motores de imanes permanentes (IP) se caracterizan por tener altas eficiencias de operación para un amplio rango de aplicaciones (Krishnan, 2010; De Almeida et al., 2014). En la industria de procesos químicos, en particular la nuclear, la necesidad de mayor seguridad y minimización de fuga de líquidos llevó al desarrollo de una bomba encapsulada que integra tanto la bomba como el motor en un único contenedor (McHardy et al., 1994), haciendo que la bomba esté libre de acoplamientos y sellos, que es donde las bombas tradicionales presentan fallas. Estadísticamente las fallas que se presentan en bombas convencionales, $7 \%$ corresponde a los acoplamientos, mientras que $52 \%$ a los sellos y empaques (Wallace et al., 1998). El motor incorpora imanes permanentes para lograr operar a alta eficiencia, donde los imanes se montan sobre la superficie de un rotor cilíndrico que se encuentra en un estator convencional con devanados trifásicos. El contenedor sin sellos tiene dos cápsulas de acero inoxidable, una montada en la superficie interior del estator y la otra sobre la superficie exterior del rotor. Es pertinente aclarar que la cápsula del rotor se forma por dos capas: una exterior de acero inoxidable para protección del rotor contra fluidos abrasivos y una interior de cobre, usada para generar el par de arranque del motor.

Normalmente, el motor de IP se conecta a un convertidor de frecuencia variable para asegurar que el motor gire síncronamente a cualquier velocidad de la bomba. Sin embargo, el costo del convertidor es alto, comparándolo con el motor, así que este nuevo tipo de bombas se diseñó para arrancar al conectarse de una fuente convencional de frecuencia fija. Los motores de IP con arranque en línea por sí mismos no son nuevos, en muchos trabajos se han empotrado alguna forma de jaula de ardilla e imanes en un rotor (Danilevich et al., 2008). Sin embargo, este motor en particular con arranque en línea, no es convencional, ya que emplea una simple capa conductora en el rotor, situada arriba de los imanes superficiales (figura 1).

Es difícil predecir la operación de motores de IP con arranque en línea debido a la naturaleza dinámica del proceso de sincronización, como se describe en Miller, (1984). La capa conductora produce un par de inducción cuando se conecta la fuente y acelera el rotor hacia su velocidad síncrona. Conforme el rotor se aproxima a la velocidad síncrona los imanes intentan 'enganchar' el rotor y si se tiene éxito ocurre la sincronización, entonces el motor gira como uno convencional de IP, ya que la cápsula del estator está hecha de

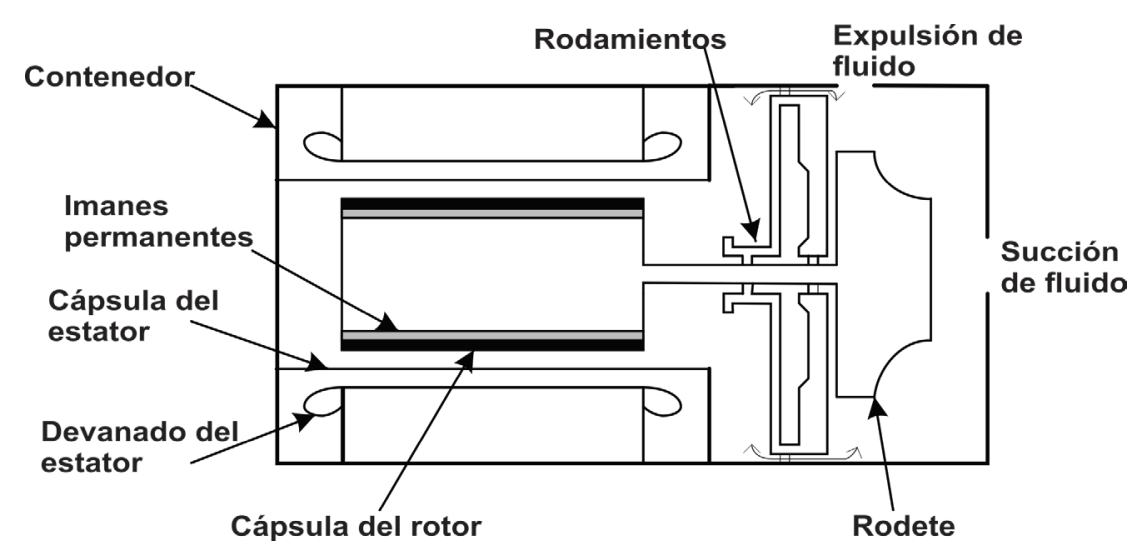

Figura 1. Bomba encapsulada con el motor y bomba integrados en un solo contenedor sin sellos 
acero inoxidable para proteger al estator de sustancias abrasivas, los imanes producen también un indeseable par de arrastre al inducir corrientes en dicha cápsula conforme el motor se acelera a partir del reposo. Por estas razones, los motores con arranque en línea presentan dificultades para sincronizarse con cargas mecánicas, con alta inercia y par de carga. Es importante que en la etapa inicial de diseño del motor se realice una predicción del par normal de inducción, el par de arrastre de inducción y el par síncrono para poder evaluar el impacto de ciertos parámetros del motor en las características operacionales, tales como la sincronización (Smith et al., 2006). Este artículo presenta una descripción detallada del desarrollo de un método analítico basado en la 'teoría de capas' para predecir los pares de inducción y síncrono en un motor encapsulado síncrono de imán permanente con arranque en línea. Se describe el desarrollo de las ecuaciones básicas electromagnéticas para el modelo por capas y se demuestra cómo puede ligarse con un circuito equivalente estándar para permitir su operación con fuente de voltaje. El modelo por capas se ha utilizado tradicionalmente desde su aparición en los trabajos de Fillimore (1956) y Cullen (1958) para predecir el comportamiento de motores de inducción, sin embargo, en este artículo se demostrará que se puede extender para predecir la operación síncrona del motor de IP, además de mostrar resultados experimentales de una bomba prototipo y el análisis del impacto de ciertas variables de diseño sobre el desempeño del motor.

\section{Modelo de capas}

Los motores convencionales de IP con arranque en línea usan una jaula de ardilla empotrada en el rotor junto con los imanes. Las corrientes inducidas en consecuencia fluyen en trayectorias bien definidas y la predicción de par, tanto dinámico como en estado estable se pueden calcular por métodos analíticos convencionales. Sin embargo, el motor de la bomba encapsulada con arranque en línea usa una cápsula conductora de cobre como devanado de inducción, en vez de una jaula de ardilla, desafortunadamente la naturaleza tridimensional de la distribución de las corrientes inducidas en la cápsula conductora no es fácil de analizar a menos que se recurra a técnicas numéricas de soluciones de campos que consumen mucho tiempo, tales como el método de elemento finito. Las técnicas analíticas normalmente se reducen a métodos bi-dimensionales utilizados por Eastham et al. (1975), Williamson et al. (1980) y Russell et al. (1958). El más prominente de estos fue el propuesto por Russell et al. (1958) que se aplica extensamente en el análisis de motores lineales para poder incluir la dirección de corriente axial de retorno usando expresiones analíticas para modificar la distribución de corriente en una hoja conductora que sobresale de la longitud del estator con excitación. Este método también es $2 \mathrm{D}$, ya que no permite la redistribución de corriente (efecto pelicular) en el espesor de la hoja conductora e ignora la influencia de la distribución de corriente sobre la densidad de flujo magnético en el entrehierro (reacción de armadura). El modelo de capas empleado en este trabajo también es 2D, adaptando tanto el efecto pelicular en la cápsula y la reacción de armadura. El método se aplica al trabajo propuesto por Russell et al. (1958), que consiste en modificar la conductividad de la cápsula para incluir el flujo de la corriente axial y cualquier saliente de la cápsula del estator como se muestra en la figura 2, lo que resulta en un método pseudo-3D.

El modelo de capas se crea en términos físicos imaginando que el motor se corta axialmente y se desenrolla hasta dejarlo plano. La formulación matemática se expresará en coordenadas rectangulares para reducir la complejidad.

El modelo en capas se muestra en la figura 3 y está sujeto a las siguientes suposiciones:

a) Las capas 2 a 5 (rotor) se mueven a velocidad $v$ respecto al estator (estacionario).

b) Todas las capas se consideran isotrópicas y homogéneas, las propiedades de los materiales se represen-

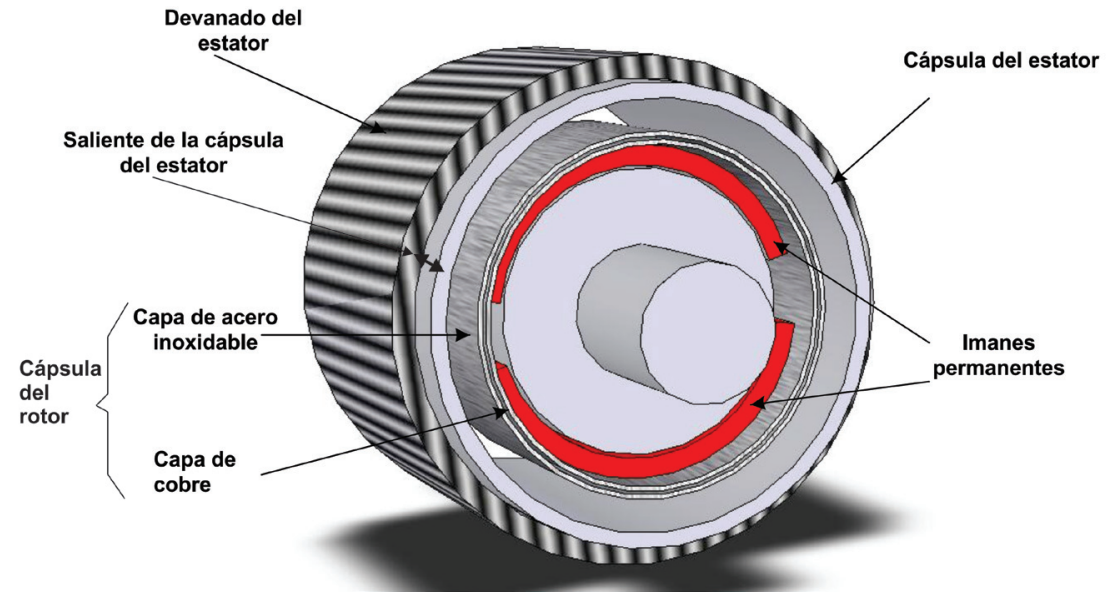

Figura 2. Esquemático que muestra el saliente de la cápsula del estator 
tan por su conductividad $\sigma$, y una permeabilidad constante $\mu$.

c) Cada imán permanente se representa por una densidad de corriente equivalente en una hoja y el ensamblado completo de imanes se considera simétrico en términos de dimensiones de los imanes, posición y espaciado.

d) El devanado de excitación del estator se reemplaza por una hoja equivalente con densidad de corriente colocada sobre la superficie interna del núcleo del estator. Esta es una técnica común en el análisis de motores.

Se asume que todas las cantidades de campo vectoriales son constantes en la dirección $y$ lo que resulta en una cantidad 2D. El flujo de corriente en la dirección $x$ se incluye usando las funciones clásicas de Russell et al. (1958) para modificar la conductividad de la cápsula conductora.

El procedimiento usual para trabajar con imanes permanentes con una curva de desmagnetización lineal es reemplazarlos con una hoja de corriente equivalente. La magnitud de la corriente se obtiene de la fuerza de coercitividad y el grosor radial del imán.
La distribución de densidad de corriente equivalente para un par de imanes con base simétrica y unitaria en $x=x_{m}$ se muestra en la figura 4 , donde $b$ es el grosor de la hoja de corriente $\mathrm{y} b \rightarrow 0$.

La fuerza magnetomotriz (FMM) efectiva está dada simplemente como (Gieras, 2009)

$F M M=H_{c} l_{m}$

donde:

$H_{c}[\mathrm{~A} / \mathrm{m}]=$ fuerza de coercitividad del imán

$l_{m} \quad=$ grosor radial de la capa del imán

La distribución de la FMM total del imán del rotor se puede expresar en una serie de armónicos de Fourier que se expresan de forma compleja como sigue

$J_{m}(x)=\sum_{k=-\infty}^{\infty} J_{m}^{k} e^{-j k x}$

donde

$k=\frac{2 p n}{d} \quad y \quad J_{m}^{k}=\left(\frac{4 p(F M M)}{\pi d l_{m}}\right) \sin \left(\frac{k \alpha}{2}\right) \sin \left(\frac{n \pi}{2}\right)$

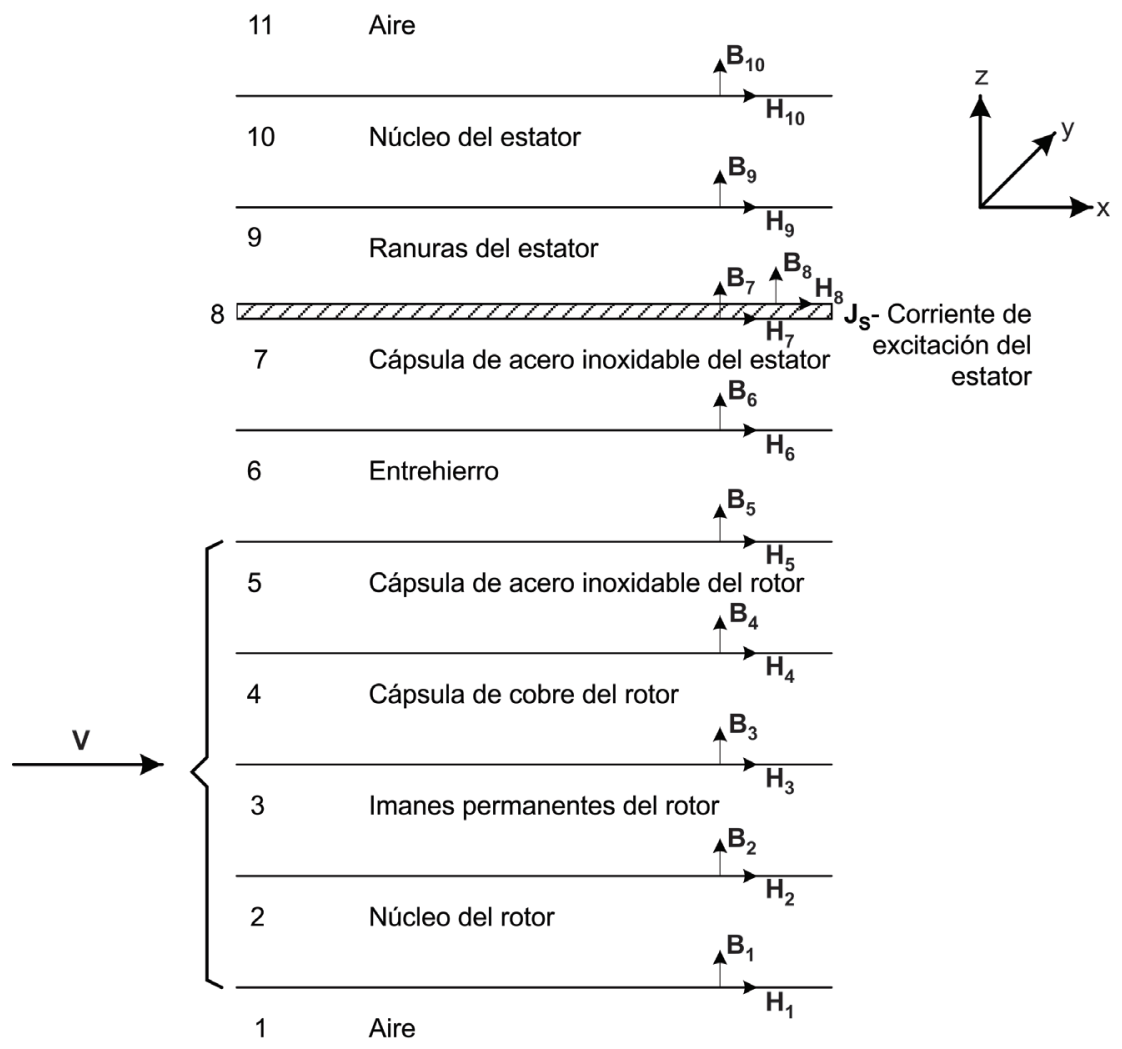

Figura 3. Modelo de capas del motor de la bomba $k=$ número de onda del armónico

$n=$ número de armónico convencional (entero)

$p=$ número de pares de polos de los imanes

$\alpha \quad$ se indica en la figura 4

$d=$ diámetro de la parte media del entrehierro

La hoja de corriente del estator se puede expresar en una serie de armónicos complejos de distribución de densidad de corriente en una forma similar a la ecuación (1), usando la corriente del devanado del estator en vez de la corriente efectiva equivalente del imán. La hoja de densidad de corriente del estator está situada entre las capas 7 y 9 y su espesor es cero. Esta puede incluirse fácilmente en el modelo de capas usando condiciones de frontera estándar para modificar las variables de campo tanto normal como tangencial. 
La solución de las ecuaciones de Maxwell en cualquier capa $n$, usando las cantidades de campo normal y tangencial $B_{z}$ y $H_{x}$ produce la siguiente solución general que se expresa en forma matricial

$$
\left[\begin{array}{c}
B_{n}+\left(\begin{array}{c}
\psi_{n} \\
\lambda_{n}
\end{array}\right) \overline{J_{k}} \\
H_{n}
\end{array}\right]=\left[\begin{array}{cc}
\cosh \lambda_{n} s_{n} & \psi_{n} \sinh \lambda_{n} s_{n} \\
\frac{1}{\psi_{n}} \sinh \lambda_{n} s_{n} & \cosh \lambda_{n} s_{n}
\end{array}\right]\left[\begin{array}{c}
B_{n-1}+\left(\frac{\psi_{n}}{\lambda_{n}}\right) \overline{J_{k}} \\
H_{n-1}
\end{array}\right]
$$

donde

$$
\begin{aligned}
J_{k} & =\begin{array}{l}
\text { cualquier } k \text {-ésimo armónico de la distribu- } \\
\\
\text { ción de corriente presente en la capa }
\end{array} \\
S_{n} & =\text { grosor radial de la capa } \\
n \text { y } n-1= & \text { los valores de los campos en la parte supe- } \\
& \text { rior e inferior de la capa } n \text {-ésima. }
\end{aligned}
$$

Las propiedades del material de la capa $n$ : permeabilidad $\mu$, y conductividad $\sigma$, junto con la frecuencia $w$, se incluyen en las constantes del campo

$\psi_{n}=j k \mu_{n} / \lambda_{n} \quad \mathrm{y} \quad \lambda_{\mathrm{n}}^{2}=k^{2}+j \omega \mu_{n} \sigma_{n}$

Las ecuaciones de campo anteriores relacionan las variables de campo $B$ y $H$ en uno de los límites o fronteras con el otro límite o frontera de la otra capa y pueden emplearse en cascada hacia arriba o hacia abajo para calcular todos los valores de las variables de campo en las fronteras a lo largo del modelo de capas mostrado en la figura 3. La solución general empieza determinando las variables de campo en las capas de excitación (estator (8) e imanes (3)). Esto se facilita si se encuentran los valores de las impedancias superficiales de campo en las fronteras de la capa de excitación, las cuales se pueden obtener empleándose en cascada, a través de las capas, en las impedancias de superficie más externas (1) y (10), donde se asume que los campos decayeron a cero conforme $Z$ tiende al infinito, usando las siguientes ecuaciones

$$
\begin{gathered}
Z_{n}=\frac{B_{n}}{H_{n}}=\frac{Z_{n-1} \cosh \lambda_{n} s_{n}+\frac{j k \mu_{n}}{\lambda_{n}} \sinh \lambda_{n} s_{n}}{\frac{\lambda_{n}}{j k \mu_{n}} Z_{n-1} \sinh \lambda_{n} s_{n}+\cosh \lambda_{n} s_{n}} \\
Z_{n-1}=\frac{B_{n-1}}{H_{n-1}}=\frac{Z_{n} \cosh \lambda_{n} s_{n}-\frac{j k \mu_{n}}{\lambda_{n}} \sinh \lambda_{n} s_{n}}{-\frac{\lambda_{n}}{j k \mu_{n}} Z_{n} \sinh \lambda_{n} s_{n}+\cosh \lambda_{n} s_{n}}
\end{gathered}
$$

Los armónicos de los campos de excitación se utilizan en cascada a través del modelo de capas y una vez que todos los campos se determinan, pueden derivarse cantidades tales como par y pérdidas de potencia del tensor de tensiones de Maxwell y el vector de Poynting (Hyat et al., 2011) respectivamente, si es que se aplican en la capa apropiada del modelo.

Una de las desventajas de este método es que cumple como solución para problemas que tienen entrada de excitación corriente, en lugar de voltaje. El modelo de capas, sin embargo, puede modificarse para emplearlo con un circuito equivalente más convencional, donde el desempeño del motor de la bomba pueda obtenerse para una fuente de voltaje constante. La figura 5 muestra el uso del modelo de capas tradicional con una fuente de corriente.

La figura 6 muestra el modelo de capas conectado a una fuente de voltaje constante en serie con la resistencia e inductancia del devanado del estator. El campo magnetizante producido por el devanado del estator ya se tomó en cuenta en el modelo de capas del motor.

Para determinar el par de inducción desarrollado durante el arranque de la bomba, el modelo de capas se usa en dos modos de estado estacionario: con solo excitación del estator y con solo excitación de los imanes del rotor. Para el primer caso, la excitación de los imanes se remueve y el modelo de capas se usa para determinar la corriente inducida en las cápsulas del estator y rotor. Los campos producidos en este modelo también se pueden usar para determinar el par de inducción que acelera el rotor. Para el segundo caso, la corriente del estator se selecciona a cero y la excitación de los imanes se aplica para determinar las corrientes producidas en la cápsula del estator y así determinar el par de inducción de arrastre. La interacción entre los imanes y el campo del estator durante el arranque produce un par oscilante en vez de un par neto de aceleración, tal que la interacción entre los imanes y el devanado del estator se utilizan solo para determinar el desempeño del motor cuando está operando en forma síncrona.

El modelo de capas se liga a la fuente de voltaje constante (figura 6) determinando la fuerza electromotriz (FEM) representada por U para una corriente unitaria inyectada en los devanados del estator del modelo de capas. Los valores de frontera de $\mathrm{B}_{\mathrm{z}} \mathrm{y}_{\mathrm{x}}$ se obtienen de las ecuaciones de la capa (1) - (6), y la FEM U se determina directamente por la densidad de flujo en la capa del devanado del estator y el número efectivo de vueltas

$\bar{U}=-w \pi d \omega \sum_{k=-\infty}^{\infty} \frac{\bar{B}_{7}^{k}}{k}$ 


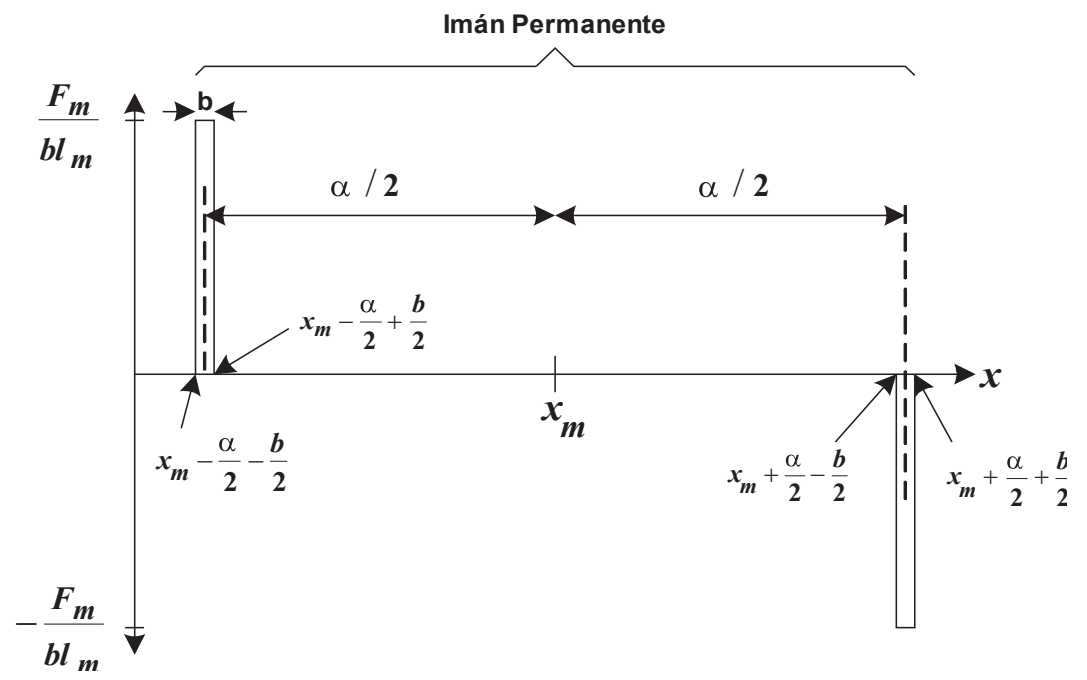

Figura 4. Densidad de corriente para un par de imanes de un par de polos

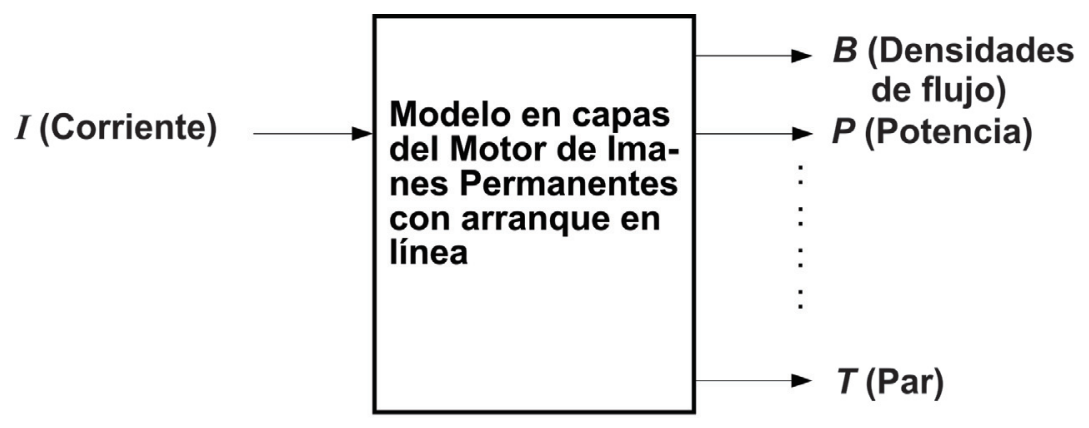

Figura5. Diagrama de la solución convencional con corriente de entrada

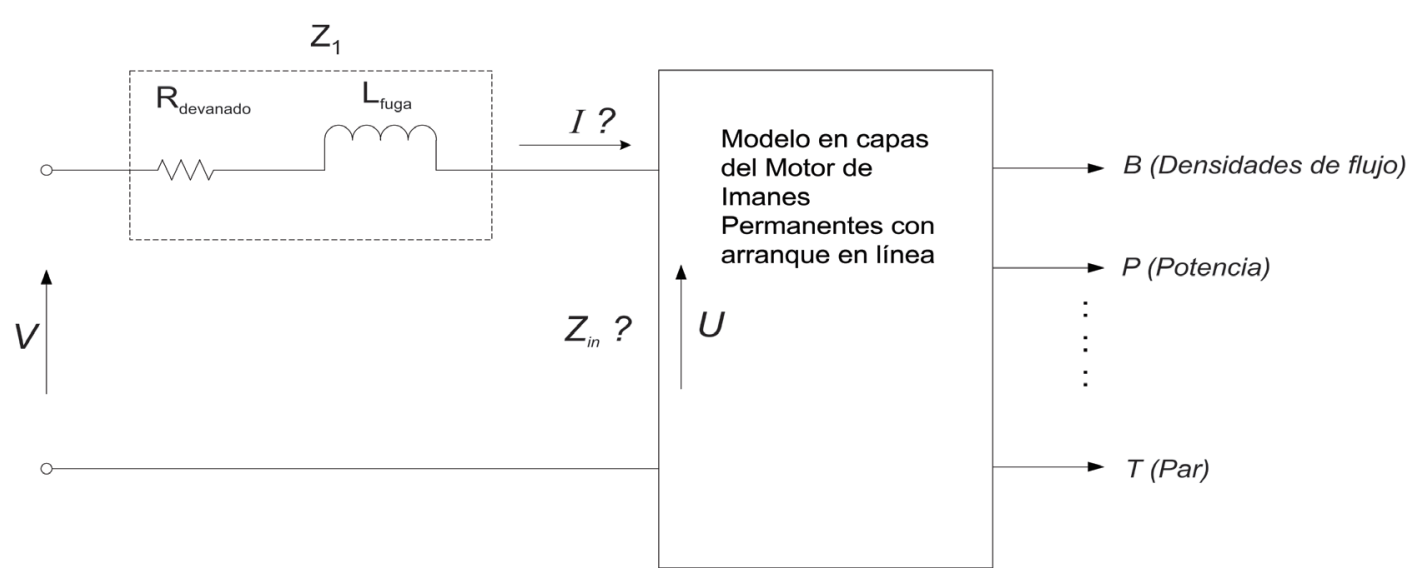

Figura 6. Diagrama modificado con una fuente de voltaje como entrada

donde

$\bar{U}=$ la FEM

$\stackrel{*}{N}^{k}=$ armónico de distribución de densidad de conductores en el estator
Ya que una corriente unitaria se inyectó en las terminales, la FEM definida en la ecuación (7) representa la impedancia de entrada al motor, donde se incluye el efecto producido por las corrientes de remolino en las cápsulas del estator y rotor. Así la impedancia del estator 
simplemente se combina con componentes externos (resistencia y reactancia del estator) para producir el valor correcto de la corriente de entrada, que a su vez, se utiliza como la entrada correcta a una segunda solución del modelo de capas para obtener todas las pérdidas y pares en el motor.

Este procedimiento no incluye los efectos de saturación. El modelo de capas puede tomar en cuenta los efectos de saturación, pero solo en sentido muy general a través de la modificación de las permeabilidades por cada capa, de forma individual. Si los efectos de saturación se incluyen, entonces el proceso mostrado anteriormente se convierte en un proceso iterativo con las permeabilidades, ya que se modifica en cada iteración hasta que la corriente de entrada converja.

Para determinar el par de arrastre producido por los imanes del rotor interactuando con la cápsula del estator, se resuelve el modelo de capas asumiendo corriente cero en el estator.

La ecuación (7) se puede emplear nuevamente para determinar la FEM inducida en el devanado del estator por los imanes permanentes, pero ahora el modelo debe resolverse usando los imanes permanentes como fuente de excitación única. Para este caso, es importante mencionar que los armónicos de campo del imán del rotor producirán fuerzas electromotrices de diferentes frecuencias.

Para la operación síncrona la FEM inducida por el rotor se usa como el fasor de referencia para seleccionar el ángulo de carga. El ángulo de fase del voltaje de estator aplicado varía respecto al ángulo de fase de la FEM $\mathrm{E}$ inducida por el rotor para representar un ángulo de carga variable. La corriente del estator se puede obtener de la diferencia entre el voltaje de estator aplicado (incluyendo el ángulo de fase para representar el ángulo de carga) y la FEM inducida por el rotor, dividida por la impedancia del estator que se obtiene del modelo de capas, sumada con la resistencia del estator y su reactancia de fuga. Esta corriente se inyecta nuevamente en el modelo de capas para proveer los valores correctos de densidades de flujo, potencias y pares. El procedimiento se repite después para diferentes ángulos de carga produciendo la curva síncrona completa de operación.

\section{Reultados experimentales}

El sistema de pruebas experimentales se compone de un prototipo de bomba encapsulada de 10kW, 2-polos, $415 \mathrm{~V}$, conectada a una máquina de CD en una configuración estándar Ward-Leonard como se muestra en la figura 7. Un transductor de par se usa para determinar el par en la flecha del motor. El motor prototipo inicialmente se probó con el estator desconectado de la fuente para determinar el par de arrastre producido por las pérdidas de las corrientes de remolino en la cápsula del estator, causadas por la rotación del imán. Es pertinente aclarar, que debido a que el motor encapsulado cuenta con arranque directo a línea, este no requiere arrancador alguno.

La figura 8 muestra las pérdidas en la cápsula predichas por el modelo de capas, que se comparan con las medidas en la flecha del motor.

Los valores teóricos de las pérdidas en la cápsula del estator son menores que los valores medidos, sin embargo, los resultados experimentales se determinaron del par de arrastre medido en la flecha del motor. Estos valores incluyen las pérdidas mecánicas rotacionales. Además, el modelo de capas no incluye las pérdidas del núcleo causadas por los campos magnéticos alternos presentes en el núcleo del estator debido al poco impacto que tienen en su operación, así que la correlación es razonable. En este punto se debe hacer notar que la magnitud de las pérdidas en la cápsula del estator es significativa y durante el diseño se debe tener especial cuidado para evitar el sobrecalentamiento del sistema contenedor de la bomba.

No fue posible realizar mediciones directas del par de inducción durante el arranque del motor debido a la presencia de grandes pares pulsantes causados por la interacción de los imanes con el campo del estator. La figura 9 sin embargo, muestra la variación de los pares síncronos teóricos y medidos con el ángulo de carga. Este es un requerimiento inusual para el modelo de capas, ya que este método tradicionalmente se ha empleado para motores de inducción. La figura 9 muestra la clásica variación sinusoidal del par síncrono respecto al ángulo de carga. El nivel de CD de par observado en la gráfica es la componente de par de arrastre producido

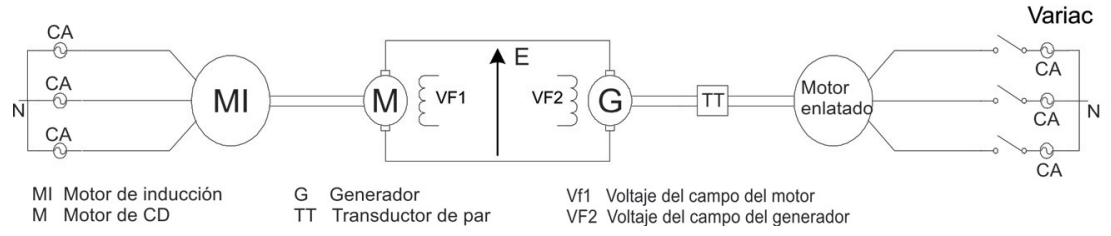

Figura 7. Sistema de pruebas experimentales 


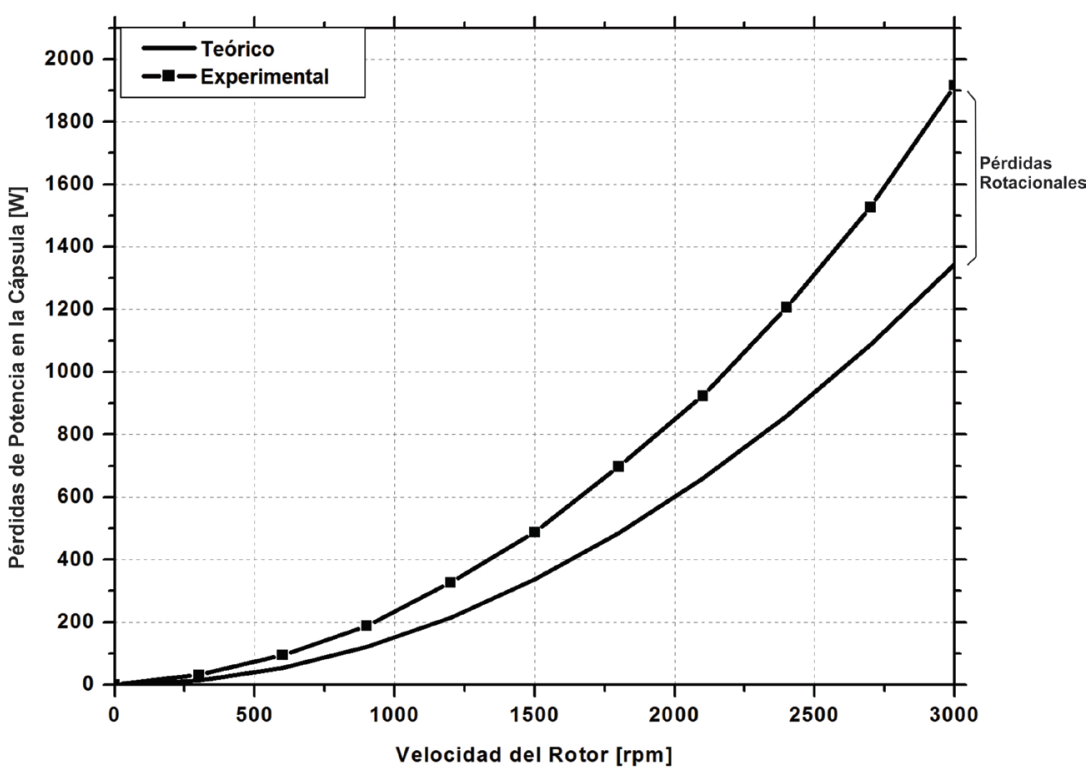

Figura 8. Pérdidas en la cápsula del estator: experimentales y teóricas

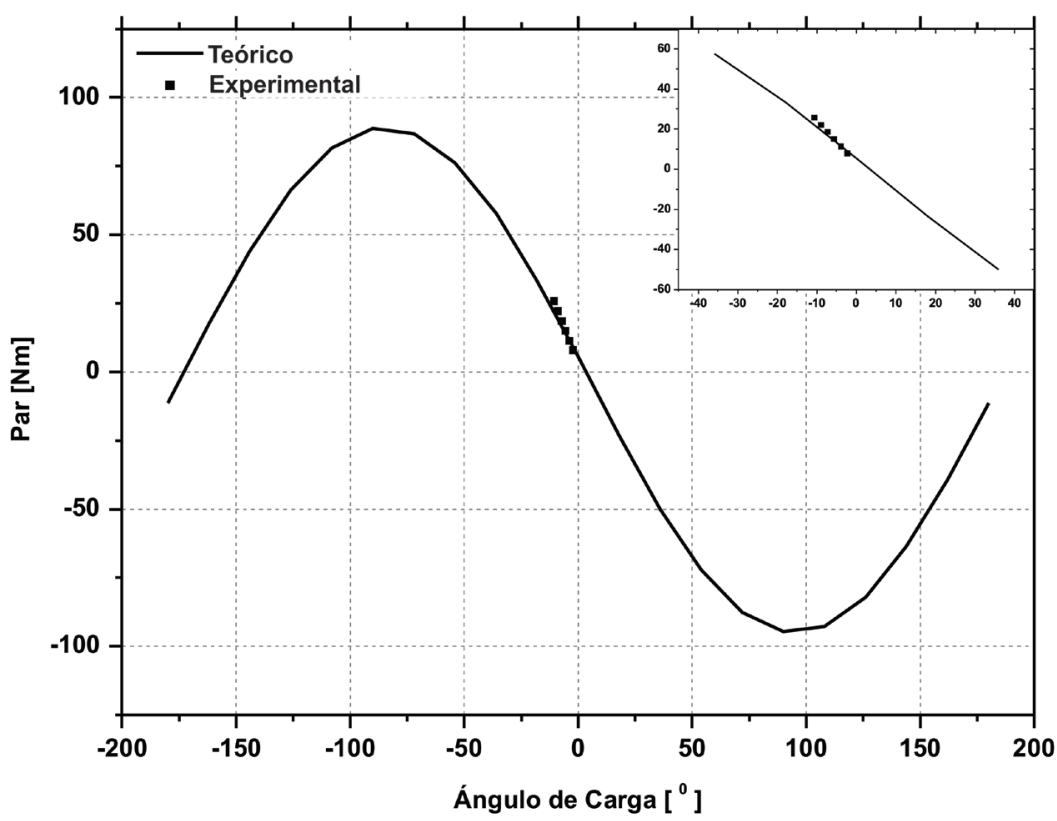

Figura 9. Par síncrono: teórico y experimental

durante la operación síncrona del motor. Este par reduce el par neto disponible en la flecha del motor.

El modelo de capas también se aplicó para investigar la variación de la corriente de entrada en función del voltaje aplicado para par de carga constante. La figura 10 muestra la correlación entre los valores teóricos y medidos de la corriente de entrada. En esta figura se nota también que hay una corriente mínima alrededor de los $370 \mathrm{~V}$ que es un valor muy próximo a la FEM E inducida por el rotor en el prototipo usado.

\section{Estudios de diseño}

El modelo de capas descrito anteriormente se destinó a una serie de estudios enfocados en varios aspectos claves de diseño del prototipo de bomba encapsulada. La figura 11 muestra la influencia de la magnitud de densidad de flujo del imán en el desempeño síncrono del motor. Como se esperaba, al reducir la magnitud del imán (1xIP, 0.75xIP, $0.5 x I P$ ) se refleja una reducción de la FEM y en consecuencia en el par.

La figura 12 muestra la influencia de la conductividad de la cápsula del rotor en el arranque del motor. El motor prototipo se diseñó con una cápsula de alta resistencia para proveer un alto par de arranque (y baja corriente) y para evitar problemas durante el arranque con líquido en la bomba, todas las curvas en la gráfica están normalizadas a este valor. Es claro que incrementando el espesor de la cápsula se reduce su resistencia y en consecuencia el par de arranque.

El uso de cápsulas con alta resistencia puede, sin embargo, reducir la velocidad del motor sin carga, lo cual podría producir problemas en la sincronización del motor. No obstante, esto no se observó en el motor prototipo, ya que fue capaz de sincronizarse fácilmente con carga e inercia nominales. La figura 12 también muestra el efecto de reemplazar la cápsula de cobre con una de aluminio, por lo que la conductividad reducida claramente aminora el par de inducción de aceleración.

La figura 13 muestra el efecto de variar el saliente de la cápsula del estator. Un factor de saliente de cero representa una cápsula de estator con una longitud axial igual a la del núcleo del estator y un factor de uno, un saliente igual a la longitud del núcleo del estator. Está claro en esta figura que las pérdidas de potencia en la cápsula y el par de arrastre resultantes aumentan conforme el factor de saliente se incremente. Sin embargo, alcanza un máximo a un factor de saliente de aproximadamente la unidad (longitud de saliente = longitud del 


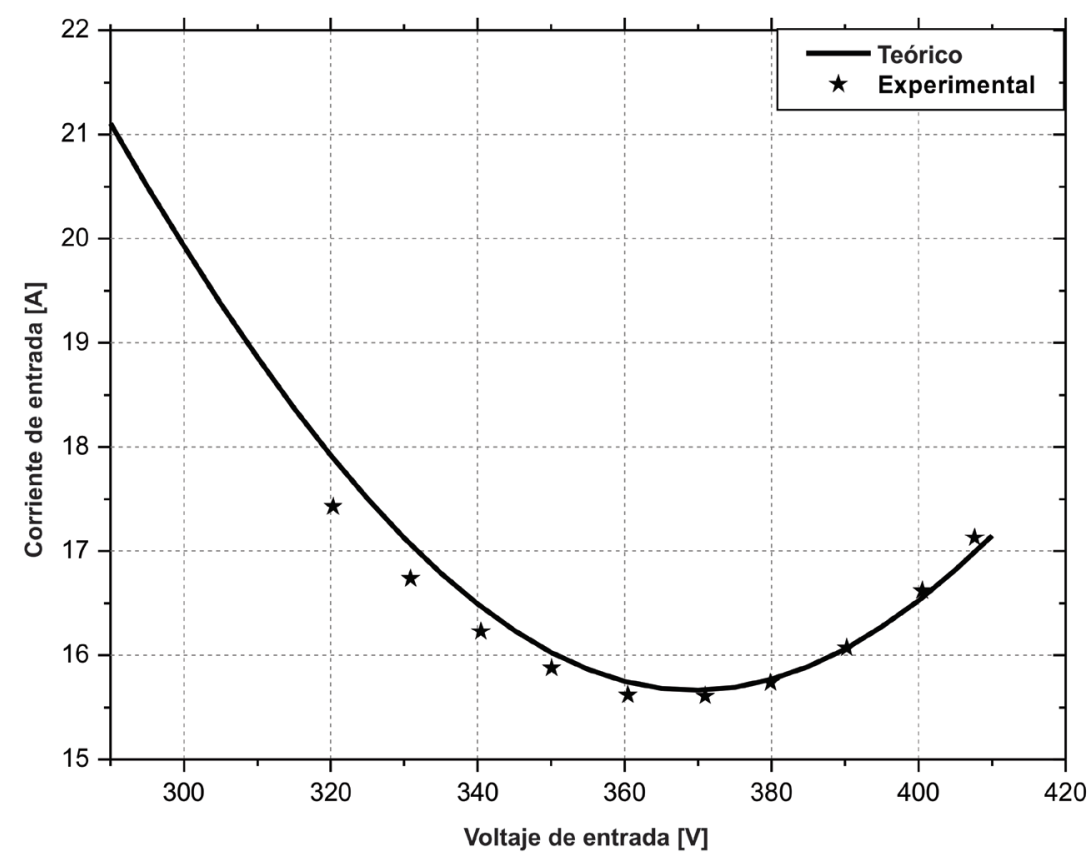

Figura 10. Corriente del estator: predicha y experimental

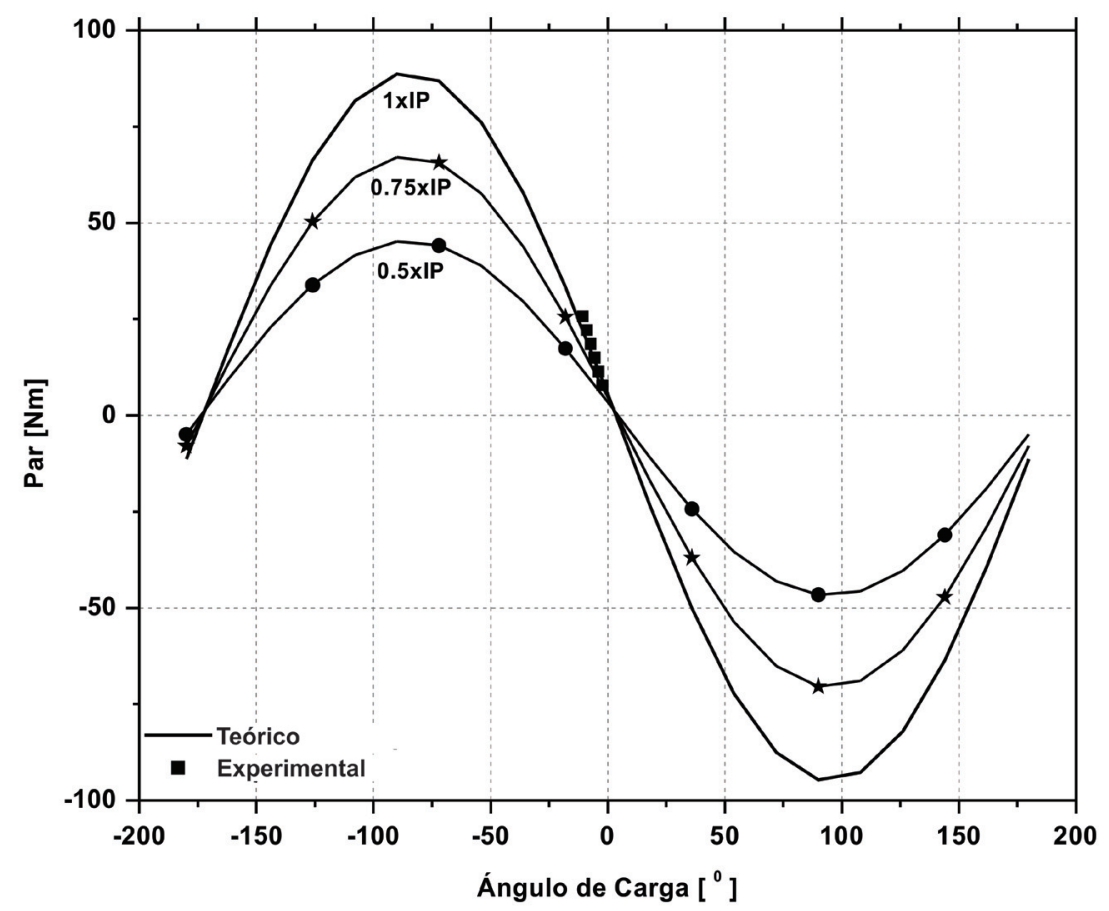

Figura 11. Par síncrono a diferentes magnitudes del campo del imán

núcleo del estator). Desde el punto de vista de diseño es claro que el saliente debería minimizarse de acuerdo con restricciones mecánicas. En el motor prototipo el factor de saliente fue de 0.6 , pero este valor fue limitado principalmente por restricciones del sistema contenedor.

La cápsula del estator utilizada en el motor prototipo tiene una alta resistencia efectiva debido a su grosor pequeño. A bajas velocidades del rotor, la frecuencia efectiva es baja y en consecuencia la impedancia de la cápsula es predominantemente resistiva y el par de arrastre del rotor es linealmente dependiente de la velocidad del rotor como se muestra en la figura 14. Sin embargo, conforme la velocidad del rotor se incrementa, la reactancia de la cápsula del rotor también y la impedancia de la cápsula del estator se domina por la inductancia de la cápsula. El efecto pelicular también se presenta a altas frecuencias y también influye en el valor de la impedancia de la cápsula. El efecto neto es que las pérdidas de la cápsula tienden a converger a un valor constante con valores muy altos de velocidad del rotor (figura 14), finalmente el par de arrastre toma la forma característica de un 'par de inducción'.

\section{Conclusiones}

El artículo demostró la operación exitosa de un nuevo tipo de bombas encapsuladas empleando un motor síncrono de imanes permanentes con arranque en línea, el cual utiliza cápsulas conductoras integradas en el sistema contenedor de líquidos. Además se demostró el desarrollo de un pseudomodelo analítico en 3D basado en la teoría de capas, donde se describió a detalle cómo se puede modificar para considerar el voltaje como entrada y predecir su respuesta síncrona. El modelo se validó con una bomba prototipo de $10 \mathrm{~kW}$ y se examinó la influencia del espesor y material de la cápsula del rotor sobre el par de aceleración. Asimismo, se aplicó una cápsula con alta resistencia en el prototipo para obtener un alto par de arranque y baja corriente. El mo- 


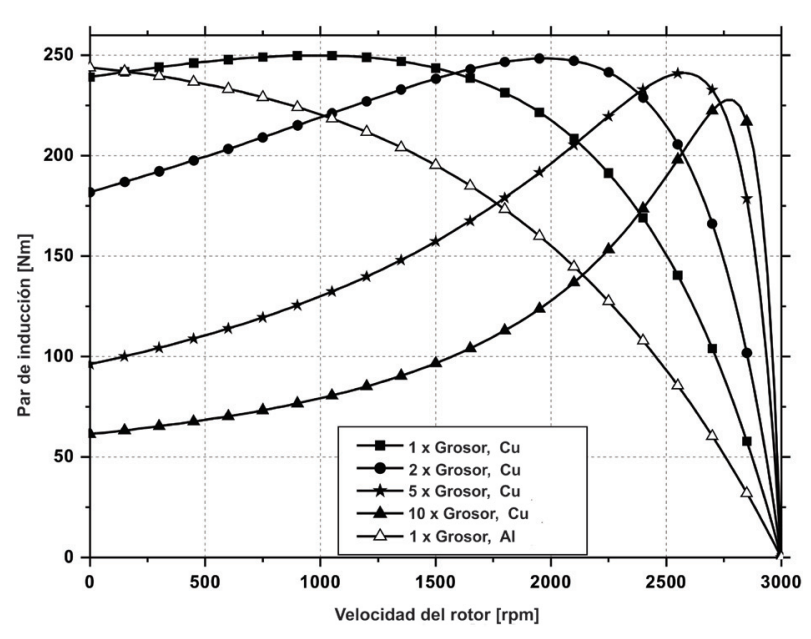

Figura 12. Variación del par de aceleración a diferentes grosores de la cápsula del rotor

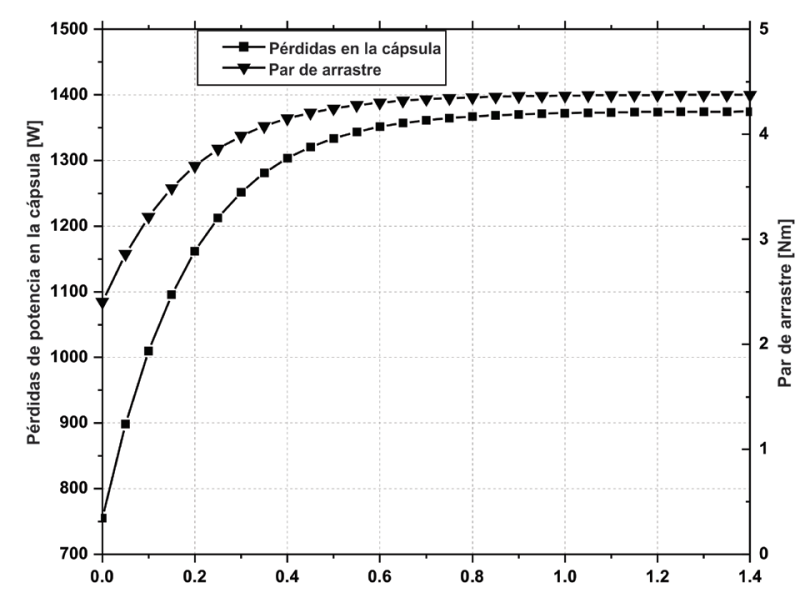

Figura 13. Variación del par de arrastre respecto al saliente

delo de capas se empleó también para examinar la influencia de los imanes sobre el desempeño síncrono del motor, además de demostrar que el saliente de la cápsula del estator debe minimizarse para reducir las pérdidas y el par de arrastre.

\section{Referencias}

Cullen A.L., Barton T.H. A simplified electro-magnetic theory of the induction motor using the concept of wave impedance. Proc. IEE, volume 105C, 1958: 331-336.

Danilevich J.B., Antipov V.N., Kruchinina I.Y., Khozikov Y.P. Design considerations of submersible unprotected solid-rotor induction motor. The 18th International Conference on Electrical Machines (ICEM), Vilamoura, Portugal, 2008, pp. 1-4.

De Almeida A.T., Ferreira J.T.E., Quintino Duarte A. Technical and economical considerations on super high-efficiency three-

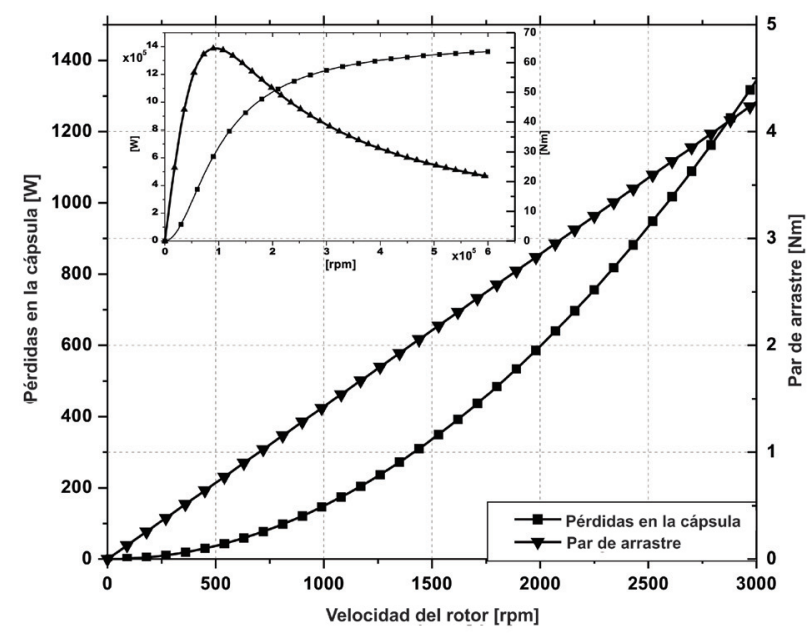

Figura 14. Pérdidas en la cápsula del estator y par de arrastre a diferentes velocidades del rotor

phase motors. IEEE Trans. on Ind. Appl., volumen 50 (número 2), marzo-abril de 2014: 1274-1285.

Eastham J.F. y Balchin M.J. Pole-change windings for linear induction motors. Proc. IEE, volumen 122, 1975: 54-160.

Fillimore R.L. Calculation of eddy-current paths in drag-cup induction motor rotors. Trans. AIEE, volumen 75, part III, 1956: 922.

Hayt W.H. y Buck. J.A. Engineering electromagnetics, 8 ed., McGraw Hill Science, 2011.

Gieras J.F. Permanent magnet motor technology: design and applications, $3^{\text {a }}$ ed., CRC Press, 2009.

Krishnan R. Permanent magnet synchronous and brushless DC motor drives, CRC Press, Taylor \& Francis Group, 2010, pp. 92-105.

McHardy S., Phillipson S.J.T. Electrical safety considerations for canned pumps in flammable material services, Fifth International Conference on Electrical Safety in Hazardous Environments, Londres, 1994, pp. 186-190.

Miller T.J.E. Synchronization of line-start permanent-magnet ac motors. IEEE Trans. on Power Apparatus and Systems, volumen PAS-103, 1984: 1822-1828.

Russell R.L., Norsworthy K.H. Eddy currents and wall losses in screened-rotor induction motors. Proc. IEE, volume 105(A), (número 163), 1958: 163-172.

Smith A.C., Peralta-Sanchez E., Purbrook S. Line-start permanent-magnet motors for pump applications. International PEMD Conference, IET, Dublin, pp. 526-531, abril de 2006.

Wallace N.M., David T.J. Pump reliability improvements through effective seals and coupling management, en: Proceedings of the $15^{\text {th }}$ International Pump Users Symposium, The Texas A\&M University System, pp. 27-40, 1998.

Williamson S., Smith A.C. Field analysis for rotating induction machines and its relationship to the equivalent-circuit method, Proc. IEE, volume 127, 1980: 83-90. 


\section{Este artículo se cita: \\ Citación estilo Chicago}

Peralta-Sánchez, Edgar, Jaime José Rodríguez-Rivas, María del Rubí Salazar-Amador. Modelo de capas para motores encapsulados de imán permanente con arranque en línea. Ingeniería Investigación y Tecnología, XVII, 01 (2016): 87-97.

\section{Citación estilo ISO 690}

Peralta-Sánchez E., Rodríguez-Rivas J.J., Salazar-Amador M.R. Modelo de capas para motores encapsulados de imán permanente con arranque en linea. Ingeniería Investigación y Tecnología, volumen XVII (número 1), enero-marzo 2016: 87-97.

\section{Semblazas de los autores}

Peralta-Sánchez Edgar. Licenciado en electrónica egresado de la Benemérita Universidad Autónoma de Puebla en 1994. Obtuvo el grado de maestro en ciencias con especialidad en ingeniería electrónica en 1999, por la Universidad de las Américas Puebla. Obtuvo el grado de PhD por la Universidad de Manchester, Inglaterra, en 2006. Actualmente trabaja como profesor investigador en la Universidad Popular Autónoma del Estado de Puebla y es miembro del SNI, nivel I.

Rodríguez-Rivas Jaime José. Ingeniero electricista egresado de la Universidad Central de Las Villas (UCLV), Cuba en 1980. Obtuvo el grado de maestro en ciencias en 1987 y el de doctor en ciencias en 1991, ambos por el Instituto Energético de Moscú. Trabajó como profesor en la UCLV desde 1980 hasta 1994. Desde 1994 a la fecha trabaja como profesor investigador en la SEPI, ESIMEZ, del IPN. Es miembro del SNI, nivel I.

Salazar-Amador María del Rubí. Licenciada en electrónica graduada en Puebla en 1994. Estudió la maestría y el doctorado en el Instituto Nacional de Astrofísica, Óptica y Electrónica, graduándose en 1996 y 2002, respectivamente. Actualmente trabaja como profesora investigadora en la Universidad Popular Autónoma del Estado de Puebla. Es miembro del SNI, nivel I. 